\title{
Scattering of Pulse Sound Signals on Elastic Body
}

\author{
A. A. Kleshchev \\ Saint - Petersburg State Navy Technical University, 190008, Saint - Petersburg, Lotsmanskaya st., 3, Russia \\ *Corresponding Author: alexalex-2@yandex.ru
}

Copyright (C) 2013 Horizon Research Publishing All rights reserved.

\begin{abstract}
With the help of the Fourier transform and of the method of the imaginary sources and imaginary scatterers is solved the problem of the scattering of the pulse sound signal by the elastic spheroidal shell, put in the plane waveguide.
\end{abstract}

Keywords Diffraction, Waveguide, Pulse, Elastic Spheroidal Shell, Source, Scatterer, Imaginary

\section{Introduction}

At the basis of the method of the imaginary sources and imaginary scatterers is solved the problem of scattering of the pulse signals by the elastic spheroidal shell, accommodated in the plane waveguide with the ideal boundary conditions. The impulse signals put the energy; therefore they are propagating with the group velocity, which lie in the principles of the method of the imaginary sources and imaginary scatterers.

\section{The Method of the Imaginary Sources and Imaginary Scatterers for the Elastic Spheroidal Shell, Put in the Plane Waveguide}

The scattering of sound by the bodies, placed in the waveguide, are investigated in the papers $[1,2,8,9,12-16]$. In the paper [2] were calculated the spectral characteristics of the ideal spheroid, placed in the sound channel, by the pulse irradiation; in the papers [2] and [8] with the help of the method of the imaginary sources and scatterers are found the vertical distributions of the scattered sound field of the ideal soft spheroid, placed in the plane waveguide, typo at the irradiation his by the harmonic signal. In the article [6] with the help of the Fourier transform and characteristics of the stationary (continuos) sound signal are calculated the pulses, scattered by the ideal prolate spheroid.

Let's put the elastic spheroidal shell into the liquid layer with the thickness $H$ and the constant sound velocity. At the upper boundary of the waveguide is fulfilled Dirichle condition, at the lower boundary - Neiman condition. The axis of the rotation of the prolate spheroidal shell will be orientated parallel to the boundaries of the waveguide and perpendicular to the plane of the Figure 1.

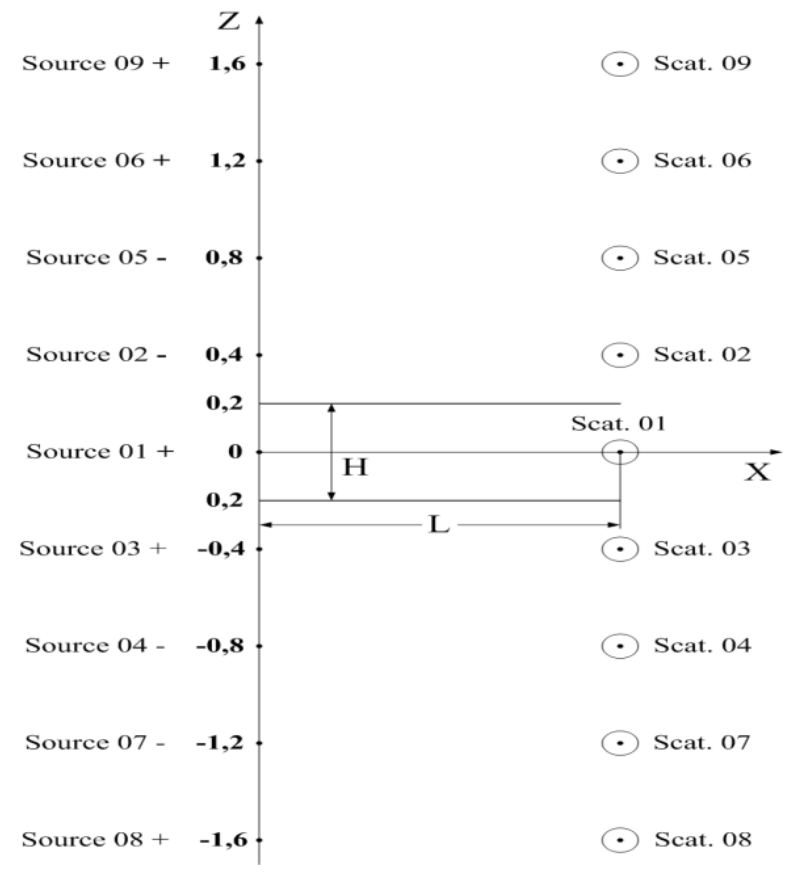

Figure 1. The mutual disposition of the pulse point-soirces and scatterers in the plane waveguide

We can do dimehsions of the scatterer, distance from it to the boundaries and the thickness of the waveguide $H$ are supposed to be such that without taking into consideration the scattering of the second order of the waves reflected from the boundaries of waveguide are not taken into account in the further process of the diffraction.

The centre of the scatterer is fixed at the distance $0,5 \cdot H$ from the bottom, at the horizontal distance $L$ from it and on the depth $0,5 \cdot H$ (Fig. 1) is placed the point-source $Q$ of the impulse sound signal. Using the method of the imaginary sources and scatterers ${ }^{[2.8]}$, are found the scattered pulse signal in the point $Q$. The sound pulse signals were the two appearances: with the harmonic and frequency-modulated filling.

The spectrum $S_{0}(2 \pi \nu)$ of the sound pulse of the source $\Psi_{i}(t)$ with the harmonic filling has the appearance [3] : 


$$
S_{0}(2 \pi v)=\frac{i v_{0}}{\pi\left(v_{0}^{2}-v^{2}\right)}(-1)^{n} \sin \left(\pi n \frac{v}{v_{0}}\right),
$$

where: $v_{0}$ - the frequency of the filling of the impulse; $n$ - the number of the oscillation periods of the harmonic signal in the pulse; $v$ - the cyclical frequency.

The spectrum $S_{0}(2 \pi v)$ is connected with $\Psi_{i}(t)$ by the inverse Fourier transform:

$$
\Psi_{i}(t)=(\pi)^{-1} \operatorname{Re} \int_{0}^{\infty} S_{0}(2 \pi v) \exp (+i 2 \pi v) d(2 \pi v)
$$

The spectrum of the reflected signal $S_{s}(2 \pi v)$ is the product of the spectrum $S_{0}(2 \pi v)$ with the corresponding angular characteristics of the scattering of the spheroidal shell $D(\eta, \varphi, v)$ ( $\eta$ and $\varphi$ - the angular coordinates of the point of the observation).

Let us consider the scatterer in the form of the elastic spheroidal shell (to Fig.. 2). All the potentials, including the plane wave potential $\Phi_{0}$, the scattered wave potential $\Phi_{1}$, the scalar shell potential $\Phi_{2}$, the Debye potentials $U$ и $V$ and the potential $\Phi_{3}$ of the gas, filling the shell, can be expanded in the spheroidal wave functions ${ }^{[8] \text { : }}$

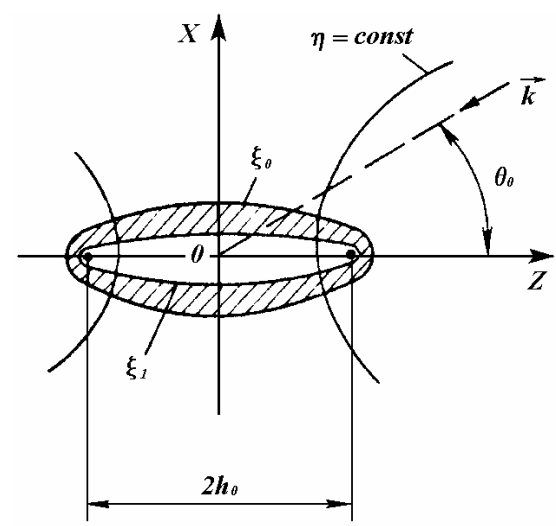

Figure 2. The elastic spheroidal shell

$$
\begin{aligned}
& \Phi_{0}=2 \sum_{m=0}^{\infty} \sum_{n \geq m}^{\infty} i^{-n} \varepsilon_{m} \bar{S}_{m, n}\left(C_{1}, \eta_{0}\right) \bar{S}_{m, n}\left(C_{1}, \eta\right) R_{m, n}^{(1)}\left(C_{1}, \xi\right) \cos m \phi ; \\
& \Phi_{1}=2 \sum_{m=0}^{\infty} \sum_{n \geq m}^{\infty} B_{m, n} \bar{S}_{m, n}\left(C_{1}, \eta\right) R_{m, n}^{(3)}\left(C_{1}, \xi\right) \cos m \phi ; \\
& \Phi_{2}=2 \sum_{m=0}^{\infty} \sum_{n \geq m}^{\infty} \bar{S}_{m, n}\left(C_{l}, \eta\right) \cos m \phi\left[C_{m, n} R_{m, n}^{(1)}\left(C_{l}, \xi\right)+D_{m, n} R_{m, n}^{(2)}\left(C_{l}, \xi\right)\right] ; \\
& \Phi_{3}=2 \sum_{m=0}^{\infty} \sum_{n \geq m}^{\infty} E_{m, n} R_{m, n}^{(1)}\left(C_{2}, \xi\right) \bar{S}_{m, n}\left(C_{2}, \eta\right) \cos m \phi ; \\
& U=2 \sum_{m=1}^{\infty} \sum_{n \geq m}^{\infty} \bar{S}_{m, n}\left(C_{t}, \eta\right) \sin m \phi\left[F_{m, n} R_{m, n}^{(1)}\left(C_{t}, \xi\right)+G_{m, n} R_{m, n}^{(2)}\left(C_{t}, \xi\right)\right] \\
& V=2 \sum_{m=0}^{\infty} \sum_{n \geq m}^{\infty} \bar{S}_{m, n}\left(C_{t}, \eta\right) \cos m \phi\left[H_{m, n} R_{m, n}^{(1)}\left(C_{t}, \xi\right)+I_{m, n} R_{m, n}^{(2)}\left(C_{t}, \xi\right)\right]
\end{aligned}
$$

where: $C_{t}=k_{2} h_{0} ; C_{1}=k h_{0}, \mathrm{k}-$ is the wave number of the sound wave in the gas, filling the shell; $B_{m, n}, C_{m, n}$, $D_{m, n}, E_{m, n}, F_{m, n}, G_{m, n}, H_{m, n}, I_{m, n}$ - are unknown expansion coefficients.

The expansion coefficients are determined from the physical boundary conditions preset at the two surfaces of the shell $\left(\xi_{0} \text { and } \xi_{1} \text {, see Fig } 2\right)^{[8]:}$

1) The continuity of the normal displacement component at both of the boundaries $\xi_{0}$ and $\xi_{1}$;

2) The identity between the normal stress in the elastic shell and the sound pressure in the liquid ( $\left.\xi_{0}\right)$ or in the gas $\left(\xi_{1}\right)$;

3) The absence of the tangential stress at both of the shell boundaries $\xi_{0}$ and $\xi_{1}$.

The corresponding expressions for the boundary conditions have the form ${ }^{[8]}$ 


$$
\begin{gathered}
\left(h_{\xi}\right)^{-1}\left(\partial \Phi_{3} / \partial \xi\right)=\left(h_{\xi}\right)^{-1}\left(\partial \Phi_{2} / \partial \xi\right)+\left(h_{\eta} h_{\phi}\right)^{-1} \times \\
\times\left.\left[(\partial / \partial \eta)\left(h_{\phi} \psi_{\phi}\right)-(\partial / \partial \phi)\left(h_{\eta} \psi_{\eta}\right)\right]\right|_{\xi=\xi_{1}} ; \\
-\lambda_{0} k^{2}\left(\Phi_{0}+\Phi_{1}\right)=-\lambda_{1} k_{1}^{2} \Phi_{2}+2 \mu_{1}\left[\left(h_{\xi} h_{\eta}\right)^{-1} \times\right. \\
\left.\times\left(\partial h_{\xi} / \partial \eta\right) u_{\eta}+\left(h_{\xi}\right)^{-1}\left(\partial u_{\xi} / \partial \xi\right)\right]\left.\right|_{\xi=\xi_{0}} ; \\
-\lambda_{2} k_{2}^{2} \Phi_{3}=-\lambda_{1} k_{1}^{2} \Phi_{2}+2 \mu_{1}\left[\left(h_{\xi} h_{\eta}\right)^{-1} \times\right. \\
\left.\times\left(\partial h_{\xi} / \partial \eta\right) u_{\eta}+\left(h_{\xi}\right)^{-1}\left(\partial u_{\xi} / \partial \xi\right)\right]\left.\right|_{\xi=\xi_{1}} ; \\
O=\left(h_{\eta} / h_{\xi}\right)(\partial / \partial \xi)\left(u_{\eta} / h_{\eta}\right)+\left.\left(h_{\xi} / h_{\eta}\right)(\partial / \partial \eta)\left(u_{\xi} / h_{\xi}\right)\right|_{\substack{\xi=\xi_{0} \\
\xi=\xi_{1}}} \\
O=\left(h_{\phi} / h_{\xi}\right)(\partial / \partial \xi)\left(u_{\phi} / h_{\phi}\right)+\left.\left(h_{\xi} / h_{\phi}\right)(\partial / \partial \phi)\left(u_{\xi} / h_{\xi}\right)\right|_{\substack{\xi=\xi_{0} \\
\xi=\xi_{1}}}
\end{gathered}
$$

Here $\lambda_{1}$ and $\mu_{1}$ - are the Lame constants of the shell material; $\lambda_{0}-$ is the bulk compression coefficient of the liquid; $\lambda_{2}-$ is the bulk compression coefficient of the gas, filling the shell ${ }^{[8]}$ :

$$
\begin{aligned}
u_{\xi} & =\left(h_{\xi}\right)^{-1}\left(\partial \Phi_{2} / \partial \xi\right) \\
& +\left(h_{\eta} h_{\varphi}\right)^{-1}\left[(\partial / \partial \eta)\left(h_{\varphi} \psi_{\varphi}\right)-(\partial / \partial \varphi)\left(h_{\eta} \psi_{\eta}\right)\right] \\
u_{\eta} & =\left(h_{\eta}\right)^{-1}\left(\partial \Phi_{2} / \partial \eta\right) \\
& +\left(h_{\xi} h_{\phi}\right)^{-1}\left[(\partial / \partial \phi)\left(h_{\xi} \psi_{\xi}\right)-(\partial / \partial \xi)\left(h_{\phi} \psi_{\phi}\right)\right] \\
u_{\phi} & =\left(h_{\phi}\right)^{-1}\left(\partial \Phi_{2} / \partial \phi\right) \\
& +\left(h_{\xi} h_{\eta}\right)^{-1}\left[(\partial / \partial \xi)\left(h_{\eta} \psi_{\eta}\right)-(\partial / \partial \eta)\left(h_{\xi} \psi_{\xi}\right)\right] .
\end{aligned}
$$

The substitution of the series ( 3 ) in the boundary conditions (4) yields the infinite system of the equations for determining the desired coefficients. Because of the orthogonality of the trigonometric functions $\cos m \varphi$ and $\sin$ $m \varphi$, the infinite system of the equations breaks into infinite subsystems with fixed numbers $m$. Each of the subsystems is solved by the truncation method. The number of the retained terms of the expansions (3) is the compound function of the wave size, of the index of the summation $m$ (the index $n$ of the retained terms is the function of the index $m$ ) and of the assigned accuracy.

The angular characteristic of elastic spheroidal shell $D(\eta, \varphi)$ can be calculated by the formula ${ }^{[8]:}$

$$
D(\eta, \varphi)=(2 / i k) \sum_{m=0}^{\infty} \sum_{n \geq m}^{\infty}(-i)^{n} B_{m, n} \bar{S}_{m, n}(C, \eta) \cos m \varphi,
$$

where: $C=k h_{0}-$ the wave dimension, $k-$ the wave number in liquid, $h_{0}-$ the half - focal distance; $\bar{S}_{m, n}(C, \eta)$ - the normalized angular spheroidal function; $R_{m, n}^{(1)}\left(C, \xi_{0}\right)$ and $R_{m, n}^{(3)}\left(C, \xi_{0}\right)-$ the radial spheroidal functions of the first and third forms correspondingly; $\varepsilon_{m}=1(m=0), 2(m \neq 0) ; \quad \xi_{0}-$ the radial coordinate of the scatterer; $\eta_{0}-$ the angular coordinate of the source.

Figure 2 shows the relative backscattering cross sections $\sigma_{0}$ for the different spheroidal bo-dies (ideal and elastic ones) and for the two angles of the irradiation $\left(\theta_{0}=0^{0}\right.$ and $\left.\theta_{0}=90^{\circ}\right)$. The Curve 1 corresponds to the steel prolate spheroidal shell, irradiated along the axis of the revolu-tion $Z\left(\theta_{0}=0^{0}\right)$. Curve 2 corresponds to the ideal soft spheroid with the same irradiation angle. Curve 3 represents the relative backscattering of the steel spheroid, irradiated at the angle $\theta_{0}=90^{\circ}$ (three - dimensional problem). Curve 4 corresponds to the rigid prolate spheroid: $\xi_{0}=1,005, \theta_{0}=90^{\circ}$; curve 5 is for the soft spheroid $\xi_{0}=1,005, \theta_{0}=90^{\circ}$. All the curves, shown in Fig. 3 represent functions of the wave size of the scatterers 
$C=k h_{0}$

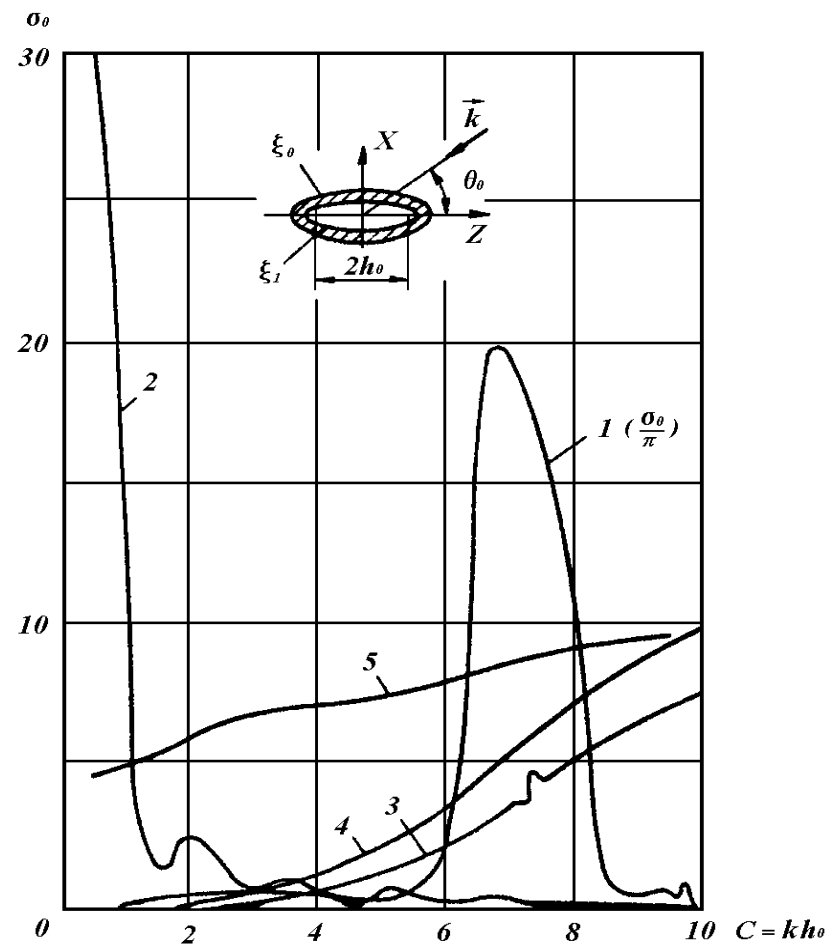

Figure 3. The relative backscattering cross sections of prolate spheroidal bodies.

\section{Conclusions}

In the paper is shown the effectiveness of the method of the imaginary sources and imaginary scatterers for the pulse sequence, got from spheroidal body and based at the use of the group velocity of the sound.

\section{Acknowledgments}

This work supported as part of research under State Contract no P242 of April 21, 2010, within the Federal Target Program "Scientific and scientific - pedagogical personnel of innovative Russia for the years 2009 - 2013".

\section{REFERENCES}

[1] A. Bostrom, In col. Artic. / Ed. by Varadan V. K., Varadan V. V., Acoustic, Electromagnetic and Elastic Wave Scattering Focus on the Matrix Approach, Pergamon press, New-York, 1980

[2] G. A. Grinblat, A. A. Kleshchev, The scattering and the emission of the sound by the bodies, placed in the waveguide, J. Techn. Acoust., 1 (1994), 3 - 5.

[3] A. A. Kharcevich, Spektrum and Analysis, GITTL, Moskau,
1957.

[4] A. A. Kleshchev, Debye and Debye - Type Potentials in Diffractions, Radiation and Elastic Waves Propagation Problems, Acoust. Phys., 58, (2012), 308 - 311.

[5] A. A. Kleshchev, Diffraction, Radiation and Propagation of Elastic Waves,Profprint, S.-Petersburg, 2006.

[6] A. A. Kleshchev, E. I. Kuznetsova, Diffraction of Impulse Sound Signals on Spheroidal Body, Put in Plane Waveguide, I.J.T.M.Ph., 2, (2012), $211-214$.

[7] A. A. Kleshchev, E. I. Kuznetsova, Interaction of Acoustic Scatterers, Acoust. Phys., 57, (2011), $308-311$.

[8] A. A. Kleshchev, Hydroacoustic Scatterers, Prima, St.Petersburg, 2012.

[9] A. A. Kleshchev, I. I. Klukin, The spectral characteristics of the scattering of the sound by the body, placed in the sound channel, Sov. Phys. Acoust., 20, (1974), 470 - 473.

[10] A. A. Kleshchev, L. S. Sheiba, The scattering of the sound wave by the ideal prolate spheroids, Sov. Phys. Acoust., 16, (1970), $264-268$

[11] A. A. Kleshchev, Scattering of Low - Frequency Pulsed Sound Signals from Elastic Cylindrical Shells, Acoust. Phys., 57, (2011), $375-380$.

[12] A. A. Kleshchev, The diffraction of the sound beam at the elastic spheroidal shell, placed in the plane waveguide and interacting with the boundaries, Proc. Symp. Interact of Acoust. Waves with Elast. Bodies, Tallinn, TGU (1989), 103 -106 .

[13] Y. A. Kravtsov, V. M. Kuzkin, V. G. Petnikov, The approximate approach to the problem of the diffraction of the waves in the waveguide with the smoothly altering parameters, News of hig. educ. inst. Radiophysics, 26, (1983), $440-446$.

[14] Y. A. Kravtsov, V. M. Kuzkin, V. G. Petnikov, The diffraction of the waves at the regular scatterers in the multimode waveguides, Sov. Phys. Acoust., 30, (1984), 339 -343 .

[15] S. O. Kvyatkovskiy, The convergency of the method of the T - matrix and Rayleigh hypo-thesis, News of hig. educ. inst. Radiophysics, 30, (1987), 1408-1410.

[16] S. O. Kvyatkovskiy, The diffraction of the sound waves at the scatterer in the waveguide, Sov. Phys. Acoust., 34, (1988), $743-745$. 